Z. GRUDZIEŃ and D. SZYNAL (Lublin)

\title{
CHARACTERIZATIONS OF UNIFORM AND EXPONENTIAL DISTRIBUTIONS VIA MOMENTS OF THE $k$ TH RECORD VALUES RANDOMLY INDEXED
}

Abstract. We characterize uniform and exponential distributions via moments of the $k$ th record statistics. Too and Lin's (1989) results are contained in our approach.

1. Introduction and preliminaries. Too and Lin (1989) characterized uniform distributions in terms of moments of order statistics and exponential distributions via moments of record values. From those results it follows that the uniform distribution can be characterized by an equality involving only two moments of order statistics, and similarly the exponential distribution can be characterized by an equality involving only two moments of record values. We characterize both those distributions (uniform and exponential) by moments of the $k$ th record values. As particular cases we get the characterization equalities quoted above.

Let $\left\{X_{n}, n \geq 1\right\}$ be a sequence of i.i.d. random variables with a common distribution function $F$. The $j$ th order statistic of a sample $\left(X_{1}, \ldots, X_{n}\right)$ is denoted by $X_{j: n}$. For a fixed integer $k \geq 1$ we define (cf. [1]) the sequence $\left\{Y_{n}^{(k)}, n \geq 1\right\}$ of $k$ th record values as follows:

$$
Y_{n}^{(k)}=X_{L_{k}(n): L_{k}(n)+k-1}, \quad n=1,2, \ldots,
$$

where the sequence $\left\{L_{k}(n), n \geq 1\right\}$ of $k$ th record times is given by

$$
\begin{aligned}
& L_{k}(1)=1, \\
& L_{k}(n+1)=\min \left\{j: j>L_{k}(n), X_{j: j+k-1}>X_{L_{k}(n): L_{k}(n)+k-1}\right\}, \\
& n=1,2, \ldots
\end{aligned}
$$

1991 Mathematics Subject Classification: 62E10, 60E99.

Key words and phrases: sample, order statistics, record values, uniform, exponential, Weibull distributions. 
We see that for $k=1$ the sequence $\left\{Y_{n}^{(1)}, n \geq 1\right\}$ is the commonly used sequence $\left\{X_{L(n)}, n \geq 1\right\}$ of record values of the sequence $\left\{X_{n}, n \geq 1\right\}$. Moreover, we note that $Y_{1}^{(n)}=\min \left(X_{1}, \ldots, X_{n}\right)=X_{1: n}$ is the minimal statistic of the sample $\left(X_{1}, \ldots, X_{n}\right)$.

2. A characterization of the uniform distribution. This section contains characterizations of the uniform distribution via moments of the $k$ th record values with both fixed and random indices. We start with the following result.

Theorem 1. Let $\left\{X_{n}, n \geq 1\right\}$ be a sequence of i.i.d. random variables with a common distribution function $F$, and assume $E\left|\min \left(X_{1}, \ldots, X_{k}\right)\right|^{2 p}$ $<\infty$ for a fixed integer $k \geq 1$ and some $p>1$. Suppose that $N$ is a positive integer-valued random variable independent of $\left\{X_{n}, n \geq 1\right\}$ such that

$$
E\left[\Gamma^{1 / q}((N-1) q+1) /(N-1) !\right]<\infty,
$$

where $q=p /(p-1)$. Then $F(x)=x^{1 / m}, x \in(0,1)$, for a positive integer $m$, iff

$$
\begin{aligned}
E\left(Y_{N}^{(k)}\right)^{2}-2 \sum_{l=0}^{m}\left(\begin{array}{c}
m \\
l
\end{array}\right)(-1)^{l} E & \left(\frac{k}{k+l}\right)^{N} Y_{N}^{(k+l)} \\
& +\sum_{l=0}^{2 m}\left(\begin{array}{c}
2 m \\
l
\end{array}\right)(-1)^{l} E\left(\frac{k}{k+l}\right)^{N}=0 .
\end{aligned}
$$

Let $F^{-1}(t)=\inf \{x: F(x) \geq t\}, t \in(0,1)$. It is known (cf. [2]) that

$$
E\left(Y_{n}^{(k)}\right)^{l}=\frac{k^{n}}{(n-1) !} \int_{0}^{1}\left(F^{-1}(t)\right)^{l}(1-t)^{k-1}[-\log (1-t)]^{n-1} d t .
$$

First consider non-random $N$ (as in Theorem 2 below). Observe that the left hand side of (2.2) in that case equals

$$
a(n):=\frac{k^{n}}{(n-1) !} \int_{0}^{1}\left(F^{-1}(t)-t^{m}\right)^{2}(-\log (1-t))^{n-1}(1-t)^{k-1} d t
$$

since

$$
\begin{aligned}
\sum_{l=0}^{m}\left(\begin{array}{c}
m \\
l
\end{array}\right)(-1)^{l} E & \left(\frac{k}{k+l}\right)^{n} Y_{n}^{(k+l)} \\
& =\frac{k^{n}}{(n-1) !} \int_{0}^{1} F^{-1}(t) t^{m}(-\log (1-t))^{n-1}(1-t)^{k-1} d t
\end{aligned}
$$


and

$$
\sum_{l=0}^{2 m}\left(\begin{array}{c}
2 m \\
l
\end{array}\right)(-1)^{l}\left(\frac{k}{k+l}\right)^{n}=\frac{k^{n}}{(n-1) !} \int_{0}^{1} t^{2 m}(-\log (1-t))^{n-1}(1-t)^{k-1} d t .
$$

Hence, by independence of $N$ and $X$ 's, (2.2) is equivalent to

$$
\sum_{n=1}^{\infty} a(n) P[N=n]=0
$$

which is equivalent to $F(x)=x^{1 / m}, x \in(0,1)$.

For the case $m=1$, Theorem 1 gives the following characterization of the uniform distribution. We have $F(x)=x, x \in(0,1)$, iff

$$
\begin{aligned}
& E\left(Y_{N}^{(k)}\right)^{2}-2\left[E Y_{N}^{(k)}-E\left(\frac{k}{k+1}\right)^{N} Y_{N}^{(k+1)}\right] \\
&+1-2 E\left(\frac{k}{k+1}\right)^{N}+E\left(\frac{k}{k+2}\right)^{N}=0 .
\end{aligned}
$$

In the case $k=1$ we get from Theorem 1 the following characterizing conditions of the uniform distribution by moments of record values.

TheOrem $1^{\prime}$. Let $\left\{X_{n}, n \geq 1\right\}$ be a sequence of i.i.d. random variables with a common distribution function $F$ such that $E\left|X_{1}\right|^{2 p}<\infty$ for some $p>1$. Suppose that $N$ is a positive integer-valued random variable independent of $\left\{X_{n}, n \geq 1\right\}$ such that $(2.1)$ is satisfied. Then $F(x)=x^{1 / m}$, $x \in(0,1)$, for some positive integer $m \geq 1$, iff

$$
\begin{aligned}
E X_{L(N)}^{2}-2 \sum_{l=0}^{m}\left(\begin{array}{c}
m \\
l
\end{array}\right)(-1)^{l} E\left(\frac{1}{1+l}\right)^{N} X_{L(N): L(N)+l} & \\
& +\sum_{l=0}^{2 m}\left(\begin{array}{c}
2 m \\
l
\end{array}\right)(-1)^{l} E\left(\frac{1}{1+l}\right)^{N}=0 .
\end{aligned}
$$

We note that $F(x)=x, x \in(0,1)$, iff

$$
E X_{L(N)}^{2}-2 E X_{L(N)}+E 2^{-N+1} X_{L(N): L(N)+1}-E 2^{-N+1}+E 3^{-N}+1=0 .
$$

Characterization conditions of the uniform distribution via moments of the $k$ th record values $Y_{n}^{(k)}$, i.e. when $P[N=n]=1$, are given in the following statements.

Theorem 2. Let $\left\{X_{n}, n \geq 1\right\}$ be a sequence of i.i.d. random variables with a common distribution function $F$ such that $E\left|\min \left(X_{1}, \ldots, X_{k}\right)\right|^{2 p}<\infty$ for a fixed integer $k \geq 1$ and some $p>1$. Then $F(x)=x^{1 / m}, x \in(0,1)$, for 
a positive integer $m$, iff

$$
\begin{aligned}
E\left(Y_{n}^{(k)}\right)^{2}-2 \sum_{l=0}^{m}\left(\begin{array}{c}
m \\
l
\end{array}\right)(-1)^{l}\left(\frac{k}{k+l}\right)^{n} E Y_{n}^{(k+l)} & \\
& +\sum_{l=0}^{2 m}\left(\begin{array}{c}
2 m \\
l
\end{array}\right)(-1)^{l}\left(\frac{k}{k+l}\right)^{n}=0 .
\end{aligned}
$$

In the case $n=1$ the condition

$$
E X_{1: k}^{2}-2\left(\begin{array}{c}
k+m \\
m
\end{array}\right)^{-1} E X_{1+m: k+m}+\left(\begin{array}{c}
k+2 m \\
k
\end{array}\right)^{-1}=0
$$

characterizes $F(x)=x^{1 / m}, x \in(0,1)$, when $E X_{1: k}^{2}<\infty$ (cf. [6]).

Hence we get the statement (cf. [4], [6]) that the uniform distribution on $(0,1)$ can be characterized by the set $\left\{E X_{1}^{2}, E X_{2: 2}\right\}$, more precisely, by the equality $E X_{2: 2}-E X_{1}^{2}=1 / 3$.

Note that $F(x)=x, x \in(0,1)$, iff

$$
\begin{aligned}
E\left(Y_{n}^{(k)}\right)^{2}-2\left(E Y_{n}^{(k)}-\left(\frac{k}{k+1}\right)^{n} E Y_{n}^{(k+1)}\right) & \\
& -2\left(\frac{k}{k+1}\right)^{n}+\left(\frac{k}{k+2}\right)^{n}+1=0 .
\end{aligned}
$$

For $n=1$,

$$
E X_{1: k}^{2}-\frac{2}{k+1} E X_{2: k+1}+\frac{2}{(k+1)(k+2)}=0
$$

iff $F(x)=x, x \in(0,1)$ (cf. [6]).

Characterization conditions of the uniform distribution via moments of $X_{L(n)}$ are as follows.

TheOrem $2^{\prime}$. Let $\left\{X_{n}, n \geq 1\right\}$ be a sequence of i.i.d. random variables with a common distribution function $F$ such that $E\left|X_{1}\right|^{2 p}<\infty$ for some $p>1$. Then $F(x)=x^{1 / m}, x \in(0,1)$, for a positive integer $m$, iff

$$
\begin{array}{rl}
E X_{L(n)}^{2}-2 \sum_{l=0}^{m}\left(\begin{array}{c}
m \\
l
\end{array}\right)(-1)^{l}\left(\frac{1}{1+l}\right)^{n} & E X_{L(n): L(n)+l} \\
& +\sum_{l=0}^{2 m}\left(\begin{array}{c}
2 m \\
l
\end{array}\right)(-1)^{l}\left(\frac{1}{1+l}\right)^{n}=0 .
\end{array}
$$

In the case $m=1$ the condition

$$
E X_{L(n)}^{2}-2 E X_{L(n)}+2^{-n+1} E X_{L(n): L(n)+1}-2^{-n+1}+3^{-n}+1=0
$$

characterizes the uniform distribution $F(x)=x, x \in(0,1)$. 
3. Characterization of the Weibull distribution. The main result of this section is contained in the following statement.

TheOREM 3. Let $\left\{X_{n}, n \geq 1\right\}$ be a sequence of i.i.d. random variables with a common distribution function $F$ and assume $E\left|\min \left(X_{1}, \ldots, X_{k}\right)\right|^{2 p}<$ $\infty$ for a fixed integer $k \geq 1$ and some $p>1$. Suppose that $N$ is a positive integer-valued random variable independent of $\left\{X_{n}, n \geq 1\right\}$. Then $F(x)=$ $1-\exp \left(-x^{1 / m}\right), x>0$, for a positive integer $m$, iff

$$
E\left(Y_{N}^{(k)}\right)^{2}-2 k^{-m} E \frac{(N+m-1) !}{(N-1) !} Y_{N+m}^{(k)}+k^{-2 m} E \frac{(N+2 m-1) !}{(N-1) !}=0
$$

provided that $E N^{2 m}<\infty, m \geq 1$.

In the case $m=1$ the condition

$$
E\left(Y_{N}^{(k)}\right)^{2}-2 k^{-1} E N Y_{N+1}^{(k)}+k^{-2} E N(N+1)=0
$$

characterizes $F(x)=1-\exp (-x), x>0$, when $E N^{2}<\infty$.

Pr o of. First consider non-random $N$. Then the left hand side of (3.1) equals

$$
\begin{aligned}
b(n):=\frac{k^{n}}{(n-1) !} \int_{0}^{1}\left(F^{-1}(t)-\left(\log \frac{1}{1-t}\right)^{m}\right)^{2} & (-\log (1-t))^{n-1}(1-t)^{k-1} d t
\end{aligned}
$$

as

$$
\begin{aligned}
E \frac{(n+m-1) !}{(n-1) ! k^{m}} Y_{n+m}^{(k)} & =\frac{k^{n}}{(n-1) !} \int_{0}^{1}\left(\log \frac{1}{1-t}\right)^{2 m+n-1}(1-t)^{k-1} d t \\
& =\frac{(n+2 m-1) !}{(n-1) ! k^{2 m}} .
\end{aligned}
$$

Hence by independence of $N$ and $X$ 's, (3.1) is equivalent to

$$
\sum_{n=1}^{\infty} b(n) P[N=n]=0,
$$

which is equivalent to $F(x)=1-\exp \left(-x^{1 / m}\right), x>0$.

For the case $k=1$ we have the following characterizing condition.

Theorem $3^{\prime}$. Let $\left\{X_{n}, n \geq 1\right\}$ be a sequence of i.i.d. random variables with a common distribution function $F$ such that $E\left|X_{1}\right|^{2 p}<\infty$ for some $p>1$, and assume that $N$ is a positive integer-valued random variable independent of $\left\{X_{n}, n \geq 1\right\}$. Then $F(x)=1-\exp \left(-x^{1 / m}\right), x \in(0, \infty)$, for 
some positive integer $m \geq 1$, iff

$$
E X_{L(N)}^{2}-2 E \frac{(N+m-1) !}{(N-1) !} X_{L(N+m)}+E \frac{(N+2 m-1) !}{(N-1) !}=0
$$

provided that $E N^{2 m}<\infty, m \geq 1$.

In particular $(m=1)$ the condition

$$
E X_{L(N)}^{2}-2 E N X_{L(N+1)}+E N(N+1)=0
$$

characterizes $F(x)=1-\exp (-x), x>0$, when $E N^{2}<\infty$.

In the case when $P[N=n]=1$ we have the following characterization conditions of the Weibull distribution.

TheOREM 4. Let $\left\{X_{n}, n \geq 1\right\}$ be a sequence of i.i.d. random variables with a common distribution function $F$ such that $E\left|\min \left(X_{1}, \ldots, X_{k}\right)\right|^{2 p}<\infty$ for a fixed integer $k \geq 1$ and some $p>1$. Then $F(x)=1-\exp \left(-x^{1 / m}\right)$, $x \in(0, \infty)$, for some positive integer $m \geq 1$, iff

$$
E\left(Y_{n}^{(k)}\right)^{2}-2 \frac{(n+m-1) !}{(n-1) ! k^{m}} E Y_{n+m}^{(k)}+\frac{(n+2 m-1) !}{(n-1) ! k^{2 m}}=0
$$

In the case $n=1$ the condition

$$
E X_{1: k}^{2}-\frac{2 m !}{k^{m}} E Y_{m+1}^{(k)}+\frac{(2 m) !}{k^{2 m}}=0
$$

characterizes $F(x)=1-\exp \left(-x^{1 / m}\right), x>0$, and for $m=1$ the condition (3.2) becomes

$$
E\left(Y_{1}^{(k)}\right)^{2}-\frac{2}{k} E Y_{2}^{(k)}+\frac{2}{k^{2}}=0,
$$

proving that each set $\left\{E\left(Y_{1}^{(k)}\right)^{2}, E Y_{2}^{(k)}\right\}, k \geq 1$, characterizes the exponential distribution.

From Theorem 4 for $k=1$ we have the following result.

Theorem $4^{\prime}$ (cf. [6]). Let $\left\{X_{n}, n \geq 1\right\}$ be a sequence of i.i.d. random variables with a common distribution function such that $E\left|X_{1}\right|^{2 p}<\infty$ for some $p>1$. Then $F(x)=1-\exp \left(-x^{1 / m}\right), x>0$, for some positive integer $m \geq 1$, iff

$$
E X_{L(n)}^{2}-2 \frac{(n+m-1) !}{(n-1) !} E X_{L(n+m)}+\frac{(n+2 m-1) !}{(n-1) !}=0 .
$$

4. Characterization conditions based on a randomization of $k$. Finally, we note that some characterization conditions in terms of moments of the minimal statistic $X_{1: K}$ (cf. [5], [3]) can be obtained from Theorem 1 and 3 , respectively, by a randomization of $k$ in the $k$ th record value (with $N=1$ a.s.). 
THEOREM 5. Let $\left\{X_{n}, n \geq 1\right\}$ be a sequence of i.i.d. random variables with a common distribution function $F$ such that $E X_{1}^{2}<\infty$ for some $p>1$. Suppose that $K$ is a positive integer-valued random variable independent of $\left\{X_{n}, n \geq 1\right\}$ with $E K<\infty$. Then $F(x)=x^{1 / m}, x \in(0,1)$, for some positive integer $m \geq 1$, iff

$$
\begin{aligned}
E X_{1: K}^{2}-2 \sum_{l=0}^{m}\left(\begin{array}{c}
m \\
l
\end{array}\right)(-1)^{l} E \frac{K}{K+l} & X_{1: K+l} \\
& +\sum_{l=0}^{2 m}\left(\begin{array}{c}
2 m \\
l
\end{array}\right)(-1)^{l} E \frac{K}{K+l}=0 .
\end{aligned}
$$

COROLlaRY. If a positive integer random variable $K$ obeys the logarithmic probability function

$$
P[K=k]=\frac{\alpha \theta^{k}}{k}, \quad k=1,2, \ldots ; 0<\theta<1, \alpha=-\frac{1}{\log (1-\theta)},
$$

then $F(x)=x^{1 / m}, x \in(0,1)$, for some positive integer $m \geq 1$, iff

$$
\begin{aligned}
E X_{1: K}^{2}-2 \sum_{l=0}^{m}\left(\begin{array}{c}
m \\
l
\end{array}\right) & (-1)^{l} E \frac{K}{K+l} X_{1: K+l} \\
& +\sum_{l=1}^{2 m}\left(\begin{array}{c}
2 m \\
l
\end{array}\right)(-1)^{l} \frac{1}{\theta^{l}}\left[1-\alpha \sum_{k=1}^{l} \frac{\theta^{k}}{k}\right]+1=0 .
\end{aligned}
$$

In the case $m=1$ we see that the condition

$$
E X_{1: K}^{2}-2\left[\alpha E X+\left(1-\frac{1}{\theta}\right) E X_{1: K}\right]+\alpha\left(\frac{3}{2}-\frac{1}{\theta}-\left(1-\frac{1}{\theta}\right)^{2} \log (1-\theta)\right)=0
$$

characterizes the uniform distribution on $(0,1)$ (cf. [5]).

Theorem 6. Let $\left\{X_{n}, n \geq 1\right\}$ be a sequence of i.i.d. random variables with a common distribution function $F$ such that $E X_{1}^{2}<\infty$ and let $K$ be a positive integer-valued random variable independent of $\left\{X_{n}, n \geq 1\right\}$ such that $E K^{m+1}<\infty$ for a fixed $m \geq 1$. Then $F(x)=1-\exp \left(-x^{1 / m}\right), x>0$, iff

$$
E X_{1: K}^{2}-2 m ! E K^{-m} Y_{m+1}^{(K)}+(2 m) ! E K^{-2 m}=0 .
$$

For the case $m=1$ the condition

$$
E X_{1: K}^{2}-2 E K^{-1} Y_{2}^{(K)}+2 E K^{-2}=0
$$

characterizes $F(x)=1-\exp (-x), x>0$. Hence we see that the set $\left\{E X_{1: K}^{2}, E K^{-1} Y_{2}^{(K)}, E K^{-2}\right\}$ characterizes the exponential distribution, and in particular we conclude that $\left\{E X_{1}^{2}, E X_{L(2)}\right\}$ characterizes the exponential distribution (cf. [4], [6]). 


\section{References}

[1] W. Dziubdziela and B. Kopociński, Limiting properties of the $k$-th record values, Appl. Math. (Warsaw) 15 (1976), 187-190.

[2] Z. Grudzień and D. Szynal, On the expected values of $k$-th record values and associated characterizations of distributions, in: Prob. Statist. Decision Theory, Proc. 4th Pannonian Sympos. Math. Statist., Reidel, 1985, 119-127.

[3] - - - Characterizations of distributions in terms of record statistics with random index, Discuss. Math. Algebra Stochastic Methods 16 (1996), 1-9.

[4] G. D. Lin, Characterizations of continuous distributions via expected values of two functions of order statistics, Sankhyā A 52 (1990), 84-90.

[5] G. D. Lin and Y. H. Too, Characterizations of distributions via moments of order statistics when sample size is random, SEA Bull. Math. 15 (1991), 139-144.

[6] —, - Characterizations of uniform and exponential distributions, Statist. Probab. Lett. 7 (1989), 357-359.

Zofia Grudzień and Dominik Szynal

Department of Mathematics

Maria Curie-Skłodowska University

pl. M. Curie-Skłodowskiej 1

20-031 Lublin, Poland

E-mail: szynal@golem.umcs.lublin.pl szynald@plumcs11.bitnet

Received on 9.5.1996;

revised version on 2.12.1996 